\title{
Discovering Latent Spatial Invariance of Urban Wireless Data using Compression and Deep Learning
}

\author{
Weisi Guo ${ }^{1,2,3 *}$ \\ ${ }^{1}$ Cranfield University, Milton Keynes, United Kingdom \\ ${ }^{2}$ University of Warwick, Coventry, United Kingdom \\ ${ }^{3}$ Alan Turing Institute, London, United Kingdom
}

\begin{abstract}
Increasingly available high resolution geospatial wireless demand data is available from high density base stations, wireless localisation, and geo-tagged social media posts. Mapping the evolving spatiotemporal demand is critical for a wide range of infrastructure services, including future network planning and operations. However, monitoring geospatial data demand across a whole city is computationally and financially expensive. Here, we show that geospatial traffic demand data from both 0.4 million Twitter posts and 3.2 million base stations records can be compressed to spatially invariant points in London. These points correspond to major sources of human movement activity that act as either facilitators (e.g. public multi-modal transport hubs) or drivers (e.g. tourist attractions and business hubs). This demonstrates that by monitoring these spatially invariant critical points, we can obtain an accurate understanding of the human demand dynamics elsewhere in the city. Indeed, the operator which maps the dynamics between these points uncover the latent human connected dynamics embedded in complex urban ecosystems.

We use both the latest signal processing technique of Graph Fourier Transform (GFT) and a AutoDecoder inspired deep learning neural network to demonstrate spatially invariant compression and both error-free and noisy recovery. These promising results show that we can exploit the connected structure of complex cities to dramatically reduce data monitoring.
\end{abstract}

Index Terms—-social media; data compression; data analysis; neural network;

\section{INTRODUCTION}

Data-driven services are critical to future smart cities, whereby geospatial consumer demand data is used to continuously improve and adapt services. In wireless networks, demand data is critical to understanding the profitability of base station deployments, tailor user-centric services, and optimise the network load balancing using mobility patterns [1]. Whilst, we have strong engineering-based and statistical modeling of infrastructure deployment (e.g. stochastic geometry), or understanding of user demand is still limited due to the lack of geospatial data [2], [3]. In recent times, increasingly available proxy geospatial data via online social network (OSN) data sets such as Twitter and other data sets

This paper is funded by EC H2020 grant 778305 - Data Aware Wireless Networks for Internet-of-Everything (DAWN4IoE). ${ }^{*}$ Corresponding Author: weisi.guo@cranfield.ac.uk have been used successfully to measure wireless demand [4][7], understand consumer reaction to poor services [8], and improve 5G deployment [9]. Other smart city applications include tracking and quantifying crowds [10], analysing crime patterns [11], and natural disaster response [12]. In general, the feedback loop between consumer demand, human experience, and personalized service provisioning is achieved through both data and statistical models where there is a shortage of data.

\section{A. Compression of Dynamic Geospatial Data for Latent Struc- tural Inference}

It is widely acknowledged that there are a variety of overlapping latent underlying spatial structures in cities [13] which could be closely related to human mobility, cyber-physical and socioeconomic interactions, as well as social networking. Theories related to demand forecasting via spatial interaction theory goes back to the 1970s [14], but the data science or big data component of it remains an open challenge. Recent work have started to conduct urban structural inference from large data sets [15] and explore its structure properties [16] and how one part of the city influence other parts. For example, the dynamic demand in one place, could be highly connected to the time-lagged demand in other places. However, very little is understood about how this might inform monitoring and we do not yet know how to recover invariant positions that represent other places. Nor do we know the context of such places should they exist. Here, we seek to use different methods to uncover the underlying latent structure and its orthogonal points.

One obvious approach that considers the data-structure is compressed sensing (CS) [17], which compresses the data by transforming them into a sparse representation. For a data matrix $\mathbf{X}$ with $N$ data points, $K$ time-steps and $r=\operatorname{rank}(\mathbf{X})$, the main challenge lies in the unknown positions of sparse nonzeros elements, which will inevitably lead to an approximately $(N+K-r) \times r / K$ points for monitoring [18]. This is not to mention that most of the CS approaches do not guarantee the spatially invariant position of monitoring points, which is not helpful in real sensor deployment actions. To further reduce the number of sampling points, our previous work in [19] proposed a data-driven GFT sampling method, which is able 


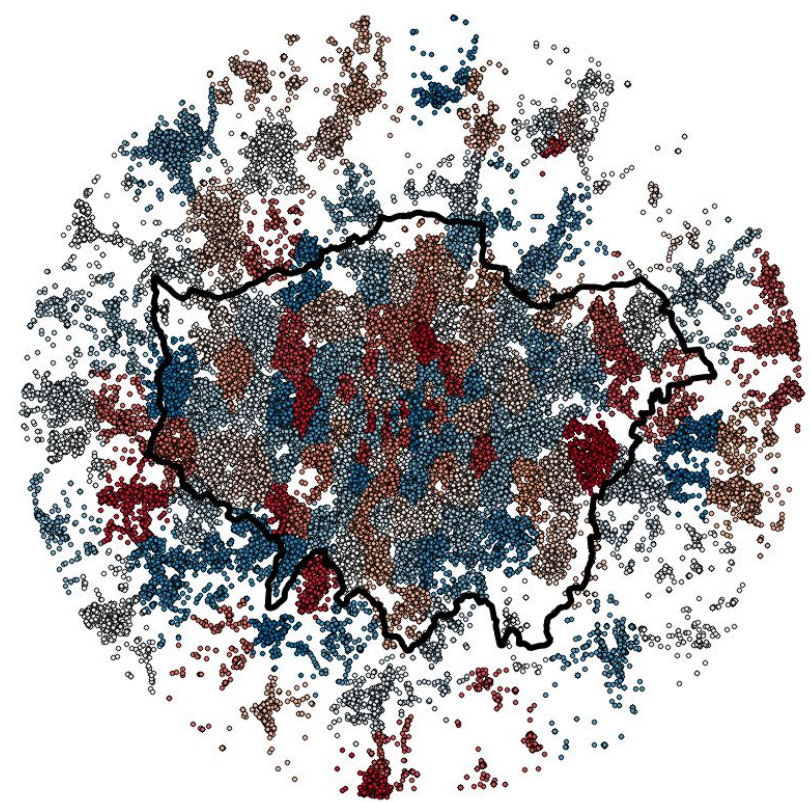

Fig. 1: Geo-tagged Tweets (0.4 million) in London with coloured clusters.

to characterize the data matrix into an $r$-bandlimited space, and thereby ensures the recovery accuracy with the monitoring of only $r$ spatially invariant orthogonal points.

\section{B. Innovation and Contribution}

In this paper, we aim to generate sparse spatially invariant monitoring points to infer the demand dynamics in all of London. We first use Graph Fourier Transform (GFT) to derive the operator that reveals the minimum number of spatially invariant monitoring points for both geo-tagged Twitter and $4 \mathrm{G}$ base station data. This enables error-free recovery of dynamics in other London locations. This approach was outlined in our recent paper [19], which minimized sensor deployment number on a dynamic complex network. Whilst we are exploiting the low-rank property, like all CS approaches, we are able to achieve a lower set of positions at the cost of losing generality, i.e., $r<(N+K-r) \times r / K$ for any $r$-rank tensor.

In this paper, we also advance this by designing a AutoDecoder $(A D)$ inspired a neural network representation of the GFT and under-sampling the process to achieve noisy recovery of dynamics in other London locations. By developing a neural network (NN), we can sample from an extremely sparse set and still recover general trends (e.g. extreme value demand spikes), significantly saving monitoring costs. Through the NN, there is a natural trade-off between reconstruction accuracy and size of the sample, and we discuss their smart city application potential.

\section{DATA \& METHOD}

\section{A. Data Set}

Our data set comprised more than 430,000 geo-tagged Tweets posted in a $40 \mathrm{~km}$ radius disc centred in Trafalgar

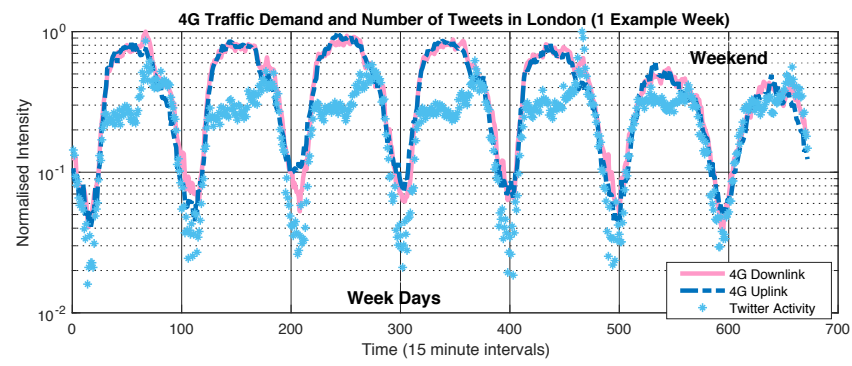

Fig. 2: 4G Traffic Demand and Number of Tweets in London (1 Example Week).

Square, London over a 2 week period - see example in Fig. 1. This data was purchased from Twitter and represents the most comprehensive geo-tagged data set for that time period (acknowledgement of open data set on Dryad [20] and was used to underpin our prior research [5]). We also have $4 \mathrm{G}$ base station data over the same period of time, comprised of 3.2 million uplink and downlink demands over 100 base stations in the same area - see example of temporal fluctuation in Fig. 2.

\section{B. Data Pre-Processing}

In order to analyse the problem (see below), we cluster the Tweets into $N$ potential sampling points. For demonstration purposes, we used k-means clustering with a well defined $k=N$ (informed by number of base stations in order to compare across data sets), but we also acknowledge that density based methods such as DBSCAN maybe more suitable. A visualization of the (clustered) Tweets posted during weekdays can be found in Fig. 1.

\section{GFT Sampling Process}

We assume that there is a latent underlying structure in cities which could be closely related to human mobility, cyberphysical and socioeconomic interactions, as well as social networking. As such, the dynamic demand in one place, could be highly connected to the time-lagged demand in other places. We seek to use Graph Fourier Transforms (GFT) to uncover the underlying latent structure and its orthogonal (spatially invariant) points. The prior work on our GFT spatially invariant sampling process is outline here [21].

We first use GFT to develop an operator that can transform the traffic demand tensor into a band-limited set of sampling points for guaranteed error-free inference performance. We then use the sampling set to hierarchically down-sample and recover the noisy dynamics via a neural network.

Graph sampling theory over a spatial domain (e.g., a map, a network, or a process with latent topological representation) aim at sampling and recovering the time-varying linearly connected signals (e.g. connection via human movement). Let the raw data tensor be $\mathbf{X} \in \mathbb{R}^{N \times K}$ which is $(r<N)$ bandlimited to a given GFT operator, denoted as $\mathbf{F}^{-1}$. That is to say: $\tilde{\mathbf{X}}=\mathbf{F}^{-1} \cdot \mathbf{X}$ has only $r$ non-zero rows. Here, we consider a fixed map with time-varying demand signals represented by the geotagged Tweets and the $4 \mathrm{G}$ base station traffic data. The $N$ rows of $\mathbf{X}$ present the time-varying signals 
$\mathrm{a}$
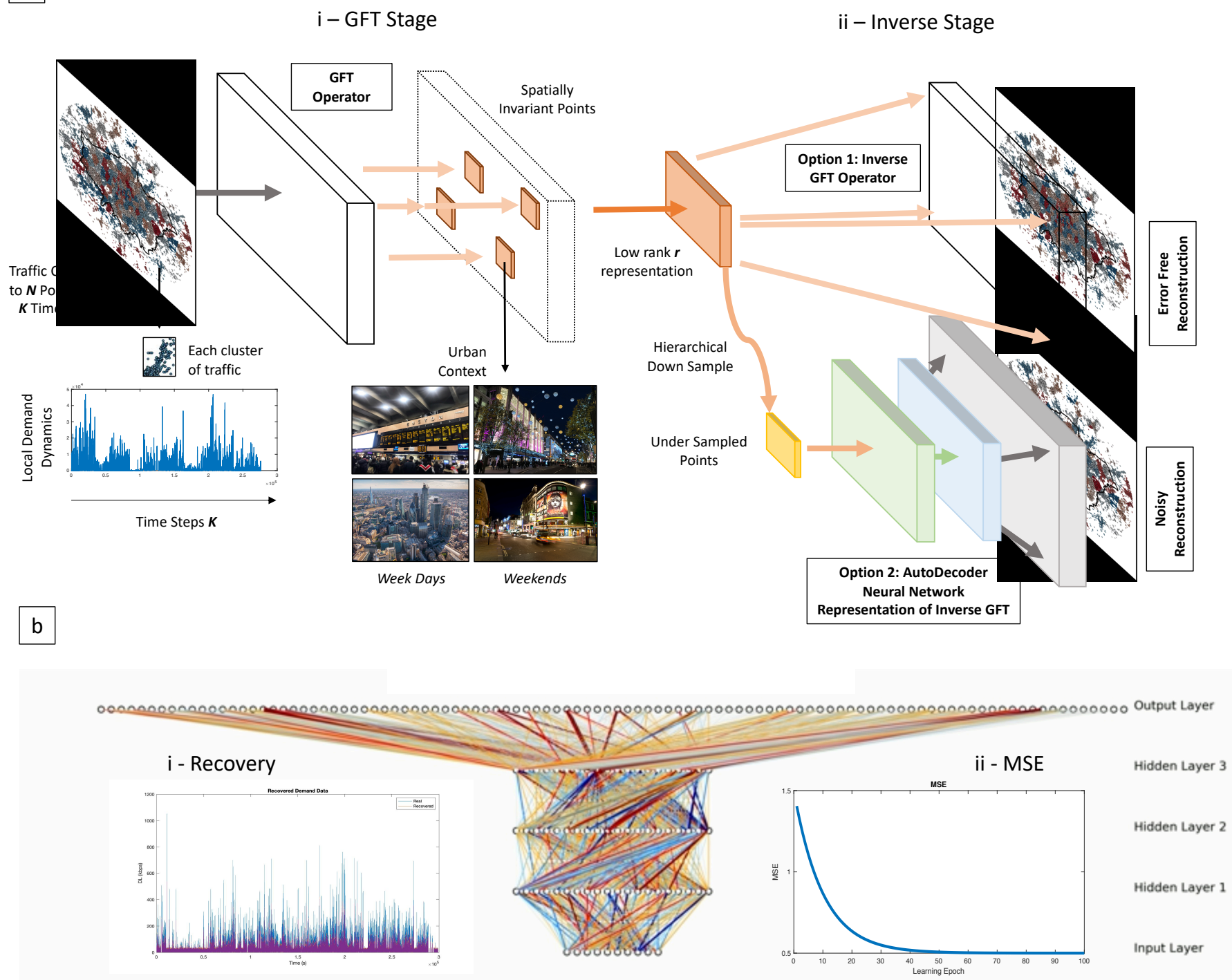

Fig. 3: Compressing dynamic demand data to spatially invariant points on map and recovering the dynamics: (a) full GFT compression and recovery by either inverse-GFT or Neural Network with hierarchical down-sampling option, and (b) AutoDecoder neural network realisation of inverse GFT with recovery dynamic example and normalised MSE tracking.

on $N$ potential sampling points (see in Fig. 1 for the $N$ clusters), and $K>N$ denotes the total number of timeindices.

We denote $\mathcal{R}$ is the set of subscripts of the nonzero rows in $\tilde{\mathbf{X}}$, and $\mathcal{V}=\{1, \cdots, N\}$. Then, we say there exists a subset $\mathcal{S} \subset \mathcal{V}$ such that:

$$
\mathbf{X}=\mathbf{F}_{\mathcal{V} \mathcal{R}} \cdot\left(\mathbf{F}_{\mathcal{S} \mathcal{R}}^{T} \cdot \mathbf{F}_{\mathcal{S R}}\right)^{-1} \cdot \mathbf{F}_{\mathcal{S} \mathcal{R}}^{T} \cdot \mathbf{X}_{\mathcal{S K}}
$$

if and only if:

$$
\operatorname{rank}\left(\mathbf{F}_{\mathcal{S R}}\right)=|\mathcal{R}|=r
$$

In Eqs. (1)-(2), $\mathbf{X}_{\mathcal{S} \mathcal{K}}$ denotes the sample of $\mathbf{X}$ from points that belongs to $\mathcal{S} . \mathbf{F}_{\mathcal{S R}}$ denotes the selection of the matrix $\mathbf{F}$ with row indices from set $\mathcal{S}$, and column indices from set $\mathcal{R}$. The sampling and recovering processes can be pursued after
(I) designing the GFT operator, and (II) the selection of $\mathcal{S}$ satisfying Eq. (2).

1) GFT Operator Design: Our design of the GFT operator (see Fig 3a-i) is based on QR factorisation: we derive the maximally linearly independent columns of $\mathbf{X}$, denoted as $\mathbf{X}_{\mathcal{V M}}=\left[\mathbf{x}_{m_{1}}, \cdots, \mathbf{x}_{m_{r}}\right]$, such that the GFT operator can be computed as:

$$
\mathbf{F}^{-1}=\mathbf{Q}^{-1}
$$

where $\mathbf{X}_{\mathcal{V M}}=\mathbf{Q} \cdot \mathbf{R}$. This $\mathbf{F}^{-1}$ ensures the $r$-bandlimited property of $\mathbf{X}$, since:

$$
\tilde{\mathbf{X}}=\mathbf{F}^{-1} \cdot \mathbf{X}
$$

2) Recovery: After the computation of the GFT operator in Eq. (3), one needs to ensure a complete recovery is to select 
$\mathcal{S}$ that satisfies Eq. (2). One can refer to [22] for details. Here, in order to achieve a robust sampling scheme on the sparse points, we consider the selection of $\mathcal{S}$ that maximizes the minimum singular of $\mathbf{F}_{\mathcal{S} \mathcal{R}}$, i.e.,

$$
\mathcal{S}_{\text {opt }}=\underset{\mathcal{S} \subset \mathcal{V}}{\operatorname{argmax}} \sigma_{\min }\left(\mathbf{F}_{\mathcal{S R}}\right),
$$

where $\sigma_{\min }(\cdot)$ denotes the smallest singular value. As such, the importance of the spatially invariant monitoring points in $\mathcal{S}$ can be ranked with the descending order of the singulars see Fig 3a-ii.

\section{AutoDecoder Neural Network Enables Hierarchical Under-Sampling \& Recovery}

The GFT in the previous subsection achieves an error-free guaranteed recovery of the demand signal in other parts of the city. However, in order to further reduce this sampling set, we can train a NN to achieve under-sampled performance. Whereas the forward GFT is performing an AutoEncoder (AE) neural network role of transforming a large data space into a sparse representation, we draw inspiration from the AutoDecoder (AD) neural network architecture, which uses sparse samples to recover back to the original data set. As such, the input layer is a set of neurons, one for each spatially invariant monitoring location found via the GFT. The input to the AE neural network is $\mathrm{N} \times \mathrm{K}$ array. The first, second, and third hidden layers are fully connected and consists of $\mathrm{N} / 2$ rectifier units, and the final layer is a linear layer with $r$ outputs. This is then reversed in the AD neural network to produce an output of all $N$ monitoring points (clusters) in the city - see Fig 3a-ii. We are inferring the time-series demand of other locations from the spatially invariant locations found via GFT. The training epoch of the network snap-shot is shown in Fig 3b, with the panel plots i) showing an example of the recovered signal in one of the unsampled points, and ii) the normalised Mean Square Error (MSE) of the recovered signal over learning epochs.

This should work, because underneath the city are latent human mobility dynamics that connect demand patterns together. The innovation here is not to optimise the overall NN architecture (e.g. via neuroevolutionary learning), but rather to demonstrate that the NN can:

1) perform equivalent inverse-GFT driven inference using the orthogonal points data and achieve near-identical error-free recovery;

2) hierarchically down-sample the critical data such that noisy recovery is possible, which is not achievable via the inverse-GFT approach.

It is worth noting that we are not performing time-series prediction (e.g. predicting next part of data using previous part, which would involve LSTM, CNN, or Gaussian Process approaches).

\section{RESULTS}

In this paper, we first cluster the 0.4 million geo-tagged Twitter posts (which have been shown to be representative
TABLE I: Spatially invariant locations in descending rank for $4 \mathrm{G}$.

\begin{tabular}{llll}
\hline Week Days & Context & Weekend & Context \\
\hline Euston & Transport & British Museum & Tourism \\
City of London & Business & Charing Cross & Tourism \\
Westminster & Government & Westminster & Tourism \\
King's Cross & Transport & Oxford Street & Shopping \\
St. Pancras & Transport & West End & Entertainment \\
Canary Warf & Business & London Eye & Tourism \\
Southwark & Business & London Bridge & Transport \\
Liverpool St. & Transport & Highbury Islington & Sport \\
Waterloo & Transport & Tower of London & Tourism \\
Victoria & Transport & St. Pancras & Transport \\
Paddington & Transport & Buckingham Palace & Tourism \\
Camden Islington & Business & & \\
Stratford & Transport & & \\
\hline
\end{tabular}

of wireless traffic [5]) to $N$ clusters, where the number of clusters is the same as the number of base stations (BSs). We then find spatially invariant clusters, as a sparse representation of all other clusters. We then show that these clusters are also the spatially invariant base stations using the 3.2 million BSs demand data set.

\section{A. Spatial Invariant Locations}

Our results in Table I for $4 \mathrm{G}$ data set show that there is a split behaviour between work days and weekends, with Fridays being closer to work day behaviour than weekend behaviour. A similar set of results were found for Twitter. We show that the following spatially invariant locations with a compression ratio of approximately $88 \%$, that is to say we only require to sample approx. 10-12 locations out of 100:

1) Week Days: Dominated by inter-city transport hubs (e.g. King's Cross, Euston, Victoria) and business hubs (e.g. Canary Warf), which demonstrates the commuting nature of London, where most of the work force travels from outside London into the city.

2) Weekends: Dominated by intra-city transport hubs (e.g. London Bridge, Stratford) and entertainment / shopping areas (e.g. West End, Oxford Street), which demonstrates the multi-cultural entertainment strength of the city, and the complete lack of business in the weekend (most of the business hubs are closed or operating at very low numbers).

The fact that other $88 \%$ places are dynamically synchronized to these spatially invariant places demonstrates the degree of approximately linear synchrony in London, whereby transportation and other activities hinge on the ability of these areas to move and attract people. For example, the rate of flow of people through a major multi-modal transport hub, entirely determines how the other locations demand services later on in time, or how a stadium attracts spectators entire determine the activity of restaurants across London before kick-off. The latent mapping between transportation hubs and activity attractors (e.g. business or entertainment) is embedded in our GFT operator and NN. 


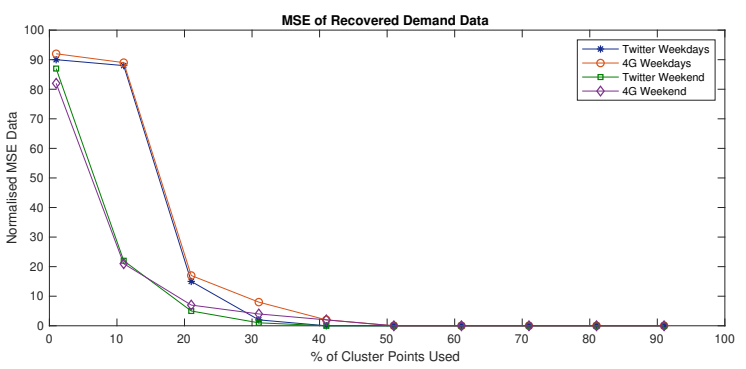

Fig. 4: Error-free recovery of demand dynamics using inverse GFT operator for Twitter and 4G telecom data during weekdays and weekend.

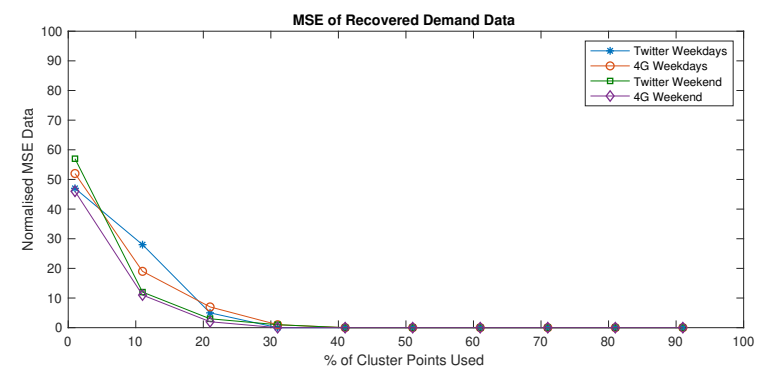

Fig. 5: Noisy recovery of demand dynamics using deep learning $\mathrm{NN}$ for Twitter and $4 \mathrm{G}$ telecom data during weekdays and weekend.

\section{B. Error-Free and Noisy Demand Recovery}

In Fig. 4 we demonstrate the error-free recovery of demand dynamics using inverse GFT operator for Twitter and 4G telecom data during weekdays and weekend. We can see that past a certain sampling point of $10-20 \%$ of points, we are guaranteed very low MSE. The arbitrarily small errors beyond that are a result of matrix operation errors for large data sets. In general the weekends require fewer sampling points than week days due to their lower rank property. In Fig. 5 we demonstrate the noisy recovery of demand dynamics using deep learning $\mathrm{NN}$ for Twitter and $4 \mathrm{G}$ telecom data during weekdays and weekend. As before, we can see that past a certain sampling point of $10-20 \%$ of points, we are guaranteed very low MSE. The arbitrarily small errors beyond that are a result of inaccurate neural network representation of the inverse GFT operator. This can be improved with optimising the $\mathrm{NN}$ architecture and activation functions using neuroevolution learning techniques, but this is beyond the scope and novelty of this paper.

\section{Uncertainty from Temporal Evolution of Urban Demand}

Despite the promising performances from both GFT and NN approaches, we are uncertain the pattern of spatially invariant monitoring points will hold over time. We use long-term $2 \mathrm{G}$ and $3 \mathrm{G}$ traffic data over a year to see how the auto-correlation within downlink (DL) and uplink (UL) traffic varies, as well as the cross-correlation (indicator of how well DL and UL are mutual indicators of each other). In Fig. 6, we can see that we fall below 50\% auto-correlation for both $2 \mathrm{G}$ and $3 \mathrm{G}$ DL and UL traffic data after 1 month, demonstrating that our

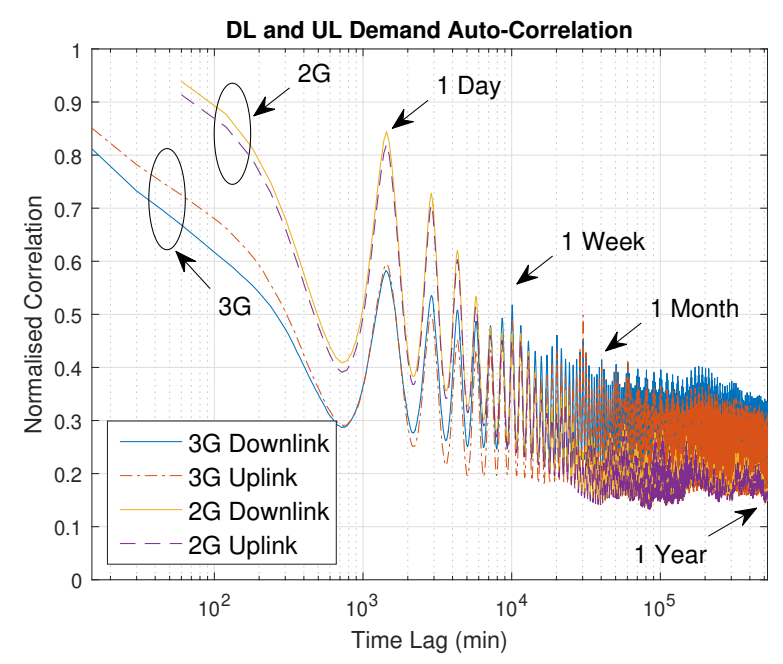

Fig. 6: Auto-correlation of DL and UL traffic in $2 \mathrm{G}$ and $3 \mathrm{G}$ over long periods

results should be re-examined after a month, or that we can only guarantee spatial invariance using weekly data for up to a month. This is the scope of further work to find long term spatial invariance, which requires long term geospatial data. This is reinforced by our earlier work in [5], where we found highly correlated spatiotemporal patterns.

\section{CONClusions AND FutURE WORK}

In this paper, we used two approaches to reconstruct demand dynamics using spatially invariant sampling points: (1) human designed GFT operator and (2) AutoDecoder inspired neural network. Here, we show that geospatial traffic demand data from both 0.4 million Twitter posts and 3.2 million base stations records can be compressed to spatially invariant points in London. These points correspond to major sources of human movement activity that act as either facilitators (e.g. public multi-modal transport hubs) or drivers (e.g. tourist attractions and business hubs). This demonstrates that by monitoring these spatially invariant critical points, we can obtain an accurate understanding of the human demand dynamics elsewhere in the city.

When conducting a sensitivity analysis using long-term proxy $2 \mathrm{G}$ and $3 \mathrm{G}$ data, we show that our results are perhaps only representative of a month. As such, we acknowledge that further work is needed to develop a more general framework that can track nonlinear changes across long term urban evolution and transfer to different cities. Nonetheless, these promising results show that we can exploit the connected structure of complex cities to dramatically reduce data monitoring.

For application and impact, we will apply this to optimize smart city services ranging from crowd management to 5G optimisation. Many of the urban applications require modeling of crowds of users [10], including safeguarding protests and festivals, tracking urban happiness [23], and quantifying consumer demand for service deployment (e.g. 5G small cells [5], [9]). Whilst research has shown that this can be accurately measured for enclosed events (e.g. 
stadiums) [10], measuring mobile crowd mobility [24], [25] in open spaces is far more challenging.

Acknowledgement: I acknowledge team members Matteo Mazzamurro, Alessio Pagani, and Zhuangkun Wei for their contribution in other papers, which helped to shape this paper.

\section{REFERENCES}

[1] A. Orsino, W. Guo, and G. Araniti, "5g multiscale mobility : A look at current and upcoming models in the next technology era," IEEE Vehicular Technology Magazine, vol. 13, no. 1, pp. 120-129, March 2018.

[2] M. Haenggi, "User point processes in cellular networks," IEEE Wireless Communications Letters, vol. 6, no. 2, pp. 258-261, April 2017.

[3] M. Mazzamurro, Y. Wu, and W. Guo, "Dynamic spatial cluster process model of geo-tagged tweets in london," IEEE International Smart Cities Conference, 2019

[4] Y. Abera and D. Hailemariam, "Spatio-temporal mobile data traffic modeling using fourier transform techniques," in 2018 International Conference on Information and Communication Technology Convergence (ICTC), Oct 2018, pp. 20-24.

[5] B. Yang, W. Guo, B. Chen, G. Yang, and J. Zhang, "Estimating mobile traffic demand using twitter," IEEE Wireless Communications Letters, vol. 5, no. 4, pp. 380-383, Aug 2016.

[6] S. Wang, X. Zhang, J. Zhang, J. Feng, W. Wang, and K. Xin, "An approach for spatial-temporal traffic modeling in mobile cellular networks," in IEEE International Teletraffic Congress, Sep. 2015, pp. 203-209.

[7] D. Lee, S. Zhou, X. Zhong, Z. Niu, X. Zhou, and H. Zhang, "Spatial modeling of the traffic density in cellular networks," IEEE Wireless Communications, vol. 21, no. 1, pp. 80-88, February 2014.

[8] W. Qi, R. Procter, J. Zhang, and W. Guo, "Mapping consumer sentiment towards wireless services using geospatial twitter data," IEEE Access, 2019.

[9] Z. Du, Y. Sun, W. Guo, Y. Xu, Q. Wu, and J. Zhang, "Data-driven deployment and cooperative self-organization in ultra-dense small cell networks," IEEE Access, vol. 6, pp. 22 839-22 848, 2018.

[10] F. Botta, H. S. Moat, and T. Preis, "Quantifying crowd size with mobile phone and twitter data," Royal Society Open Science, vol. 2, 2015.

[11] M. Gerber, "Predicting crime using twitter and kernel density estimation," Decision Support Systems, vol. 61, 2014.

[12] N. Tkachenko, R. Procter, and S. Jarvis, "Predicting the impact of urban flooding using open data," Royal Society Open Science, vol. 3, 2016.

[13] X. Zhou, A. Noulas, C. Mascolo, and Z. Zhao, "Discovering latent patterns of urban cultural interactions in wechat for modern city planning," in Proceedings of the 24th ACM SIGKDD International Conference on Knowledge Discovery \&\#38; Data Mining, ser. KDD '18. New York, NY, USA: ACM, 2018, pp. 1069-1078. [Online]. Available: http://doi.acm.org/10.1145/3219819.3219929

[14] A. Wilson, "Boltzmann, lotka and volterra and spatial structural evolution: an integrated methodology for some dynamical systems," Journal of The Royal Society Interface, vol. 5, no. 25, pp. 865-871, 2008.

[15] Y. Zhi, H. Li, D. Wang, M. Deng, S. Wang, J. Gao, Z. Duan, and Y. Liu, "Latent spatio-temporal activity structures: a new approach to inferring intra-urban functional regions via social media check-in data," Geo-spatial Information Science, vol. 19, no. 2, pp. 94-105, 2016.

[16] D. A. Griffith and K. G. Jones, "Explorations into the relationship between spatial structure and spatial interaction," Environment and Planning A: Economy and Space, vol. 12, no. 2, pp. 187-201, 1980.

[17] X. Ding, W. Chen, and I. Wassell, "Joint Sensing Matrix and Sparsifying Dictionary Optimization for Tensor Compressive Sensing," IEEE Transactions on Signal Processing, vol. 65, 2017.

[18] E. J. Candes and Y. Plan, "Tight oracle inequalities for low-rank matrix recovery from a minimal number of noisy random measurements," IEEE Transactions on Information Theory, vol. 57, no. 4, pp. 2342-2359, 2011.

[19] Z. Wei, A. Pagani, G. Fu, I. Guymer, W. Chen, J. A. McCann, and W. Guo, "Optimal sampling of water distribution network dynamics using graph fourier transform," IEEE Transactions on Network Science and Engineering, pp. 1-1, 2019.

[20] B. Yang, W. Guo, B. Chen, G. Yang, and J. Zhang, "Data from: Estimating mobile traffic demand using twitter," 2016. [Online]. Available: https://doi.org/10.5061/dryad.35m1f4q
[21] Z. Wei, A. Pagani, G. Fu, I. Guymer, W. Chen, J. A. McCann, and W. Guo, "Optimal sampling of water distribution network dynamics using graph fourier transform," IEEE Transactions on Network Science and Engineering, pp. 1-1, 2019.

[22] A. Ortega, P. Frossard, J. Kovačević, J. M. Moura, and P. Vandergheynst, "Graph signal processing: Overview, challenges, and applications," Proceedings of the IEEE, vol. 106, no. 5, pp. 808-828, 2018.

[23] A. Tsakalidis, M. Liakata, T. Damoulas, B. Jellinek, W. Guo, and A. Cristea, "Combining heterogeneous user generated data to sense well-being," in International Conference on Computational Linguistics, Osaka, Japan, Dec. 2016, pp. 3007-3018.

[24] N. Yang, X. Kong, F. Wang, and P. S. Yu, When and Where: Predicting Human Movements Based on Social Spatial-Temporal Events. SIAM, 2014, pp. 515-523. [Online]. Available: https: //epubs.siam.org/doi/abs/10.1137/1.9781611973440.59

[25] G. Chen, A. C. Viana, and M. Fiore, "Takeaways in large-scale human mobility data mining : (invited paper)," in 2018 IEEE International Symposium on Local and Metropolitan Area Networks (LANMAN), June 2018, pp. 55-60. 


\section{Discovering latent spatial invariance of urban wireless data using compression and deep learning}

Guo, Weisi

IEEE

Guo W. (2020) Discovering latent spatial invariance of urban wireless data using compression and deep learning. In: ICC 2020 - 2020 IEEE International Conference on Communications (ICC), 7-11 June 2020, Dublin https://doi.org/10.1109/ICC40277.2020.9148727

Downloaded from Cranfield Library Services E-Repository 